\title{
Die Meinung eines behandelnden Arztes
}

\section{Pierre Vallon}

Facharzt für Psychiatrie und Psychotherapie

In der Funktion als behandelnder Arzt wurde ich gebeten, mich in die Begleitgruppe des Forschungsprojekts «Die Zusammenarbeit zwischen der IV und den behandelnden Ärztinnen und Ärzten» von Christian Bolliger und Marius Féraud einzubringen.

Vorliegend einige Überlegungen zum Thema dieses Forschungsberichts aus Praktikersicht:

Die Autoren haben in einer schriftlichen Umfrage alle kantonalen IV-Stellen nach ihrem Verhältnis zu den behandelnden Ärzten befragt. Aus logistischen Gründen konnte umgekehrt nicht die gesamte Ärzteschaft der Schweiz befragt werden. Die Forscher beschränkten sich auf Ärzte aus fünf repräsentativen Kantonen (Stadt/Land, Deutschschweiz/ Romandie). Dabei zeigen sich wichtige Unterschiede, die einerseits auf den Kontext (medizinische und allgemeine Demographie, Anzahl zu behandelnder IVAnfragen) zurückzuführen sind, andererseits aber auch auf die Qualität der persönlichen Beziehungen zwischen IV-Stelle und den Ärzten aus ihrem Kanton.

Die Ärzteschaft ist aktuell nur ungenügend über den rechtlichen Rahmen und die Praxis der IV-Stellen informiert.

Die Studienresultate sind sehr interessant; es wurden insbesondere Vorschläge ausgearbeitet, um die Kontakte zwischen IV-Stellen und behandelnden Ärzten zu verbessern. Die Ärzteschaft ist aktuell nur ungenügend über den rechtlichen Rahmen und die Praxis der IVStellen informiert. Das müsste verbessert werden, speziell bei der beruflichen Eingliederung und der Suche nach einer Tätigkeit, die den funktionnellen Einschrän- kungen des Patienten Rechnung trägt. Regelmässige Treffen zwischen Vertretern der IV-Stellen und der behandelnden Ärzte könnten das theoretische Wissen erweitern und helfen, die gegenseitigen Vorurteile abzubauen. In der konkreten Fallarbeit müssten die Kommunikationsmittel an die Zeit angepasst werden. Dadurch könnte der Eindruck der behandelnden Ärzte korrigiert werden, dass die IV-Stelle eine "Black Box» sei, die nur Anfragen für Arztberichte oder Mahnun-

Es wurden insbesondere Vorschläge ausgearbeitet, um die Kontakte zwischen IV-Stellen und behandelnden Ärzten zu verbessern.

gen generiert. Soll der behandelnde Arzt für die IV tatsächlich eine unverzichtbare Informationsquelle sein, muss er auch über die verschiedenen Verfahrensschritte informiert werden, dies immer mit dem Einverständnis des Patienten. Eine Beteiligung des Arztes / der Ärztin an beruflichen Massnahmen setzt regelmässige Kontakte mit Vertretern der IV voraus.

Es ist ein besonderes Verdienst dieser Studie, dass sie dazu beiträgt, das von der IV und der Rechtsprechung gehegte Vorurteil abzubauen, der behandelnde Arzt sei parteiisch. Es kann jedoch nicht im Interesse des Arztes liegen, seinen Patienten ohne Beschäftigung auf eine IV-Rente warten zu lassen. Damit das übergeordnete Ziel, den Patienten an seinem Arbeitsplatz zu halten oder in einer angepassten Tätigkeit einzugliedern, erreicht werden kann, braucht es künftig eine bessere Zusammenarbeit zwischen IV-Stellen und behandelnden Ärzten. 